\title{
Structural and cellular features in metaphyseal and diaphyseal periosteum of osteoporotic rats
}

\author{
Wei Fan · Stefan A. W. Bouwense • \\ Ross Crawford · Yin Xiao
}

Received: 29 July 2009/Accepted: 2 March 2010/Published online: 16 March 2010

(C) The Author(s) 2010. This article is published with open access at Springerlink.com

\begin{abstract}
Despite the important physiological role of periosteum in the pathogenesis and treatment of osteoporosis, little is known about the structural and cellular characteristics of periosteum in osteoporosis. To study the structural and cellular differences in both diaphyseal and metaphyseal periosteum of osteoporotic rats, samples from the right femur of osteoporotic and normal female Lewis rats were collected and tissue sections were stained with hematoxylin and eosin, antibodies or staining kit against tartrate resistant acid phosphatase (TRAP), alkaline phosphatase (ALP), vascular endothelial growth factor (VEGF), von Willebrand (vWF), tyrosine hydroxylase $(\mathrm{TH})$ and calcitonin gene-related peptide (CGRP). The results showed that the osteoporotic rats had much thicker and more cellular cambial layer of metaphyseal periosteum compared with other periosteal areas and normal rats $(P<0.001)$. The number of $\mathrm{TRAP}^{+}$osteoclasts in bone resorption pits, $\mathrm{VEGF}^{+}$cells and the degree of vascularization were found to be greater in the cambial layer of metaphyseal periosteum of osteoporotic rats $(P<0.05)$, while no significant difference was detected in the number of $\mathrm{ALP}^{+}$cells between the two groups. Sympathetic nerve fibers identified by $\mathrm{TH}$ staining were predominantly
\end{abstract}

Wei Fan and Stefan A. W. Bouwense are the co-first authors and equal contribution to this study.

W. Fan · S. A. W. Bouwense · R. Crawford · Y. Xiao ( $\square)$ Bone Tissue Engineering, Institute of Health and Biomedical Innovation, Queensland University of Technology, Kelvin Grove Campus, Brisbane Qld 4059, Australia

e-mail: yin.xiao@qut.edu.au

S. A. W. Bouwense

UMC St Radboud, Nijmegen, The Netherlands located in the cambial layer of metaphyseal periosteum of osteoporotic rats. No obvious difference in the expression of CGRP between the two groups was found. In conclusion, periosteum may play an important role in the cortical bone resorption in osteoporotic rats and this pathological process may be regulated by the sympathetic nervous system.

Keywords Periosteum - Osteoporosis · Osteoblasts . Osteoclasts $\cdot$ Blood vessels $\cdot$ Nerve fibers

\section{Introduction}

The mineralization and development of bone tissue is a dynamic process determined by the balance between the bone formation and resorption occurring on periosteal and endosteal surfaces (Pogoda et al. 2005; Rauch et al. 2007; Seeman 2003c). This modeling and remodeling process will alter the size, shape, internal structure, total mass, and finally the mechanical strength of bone. The thickness of cortical bone, a crucial feature in determining bending strength of bone, is defined both by periosteal and endosteal bone apposition and resorption. Osteoporosis is a metabolic disorder of the bone whereby the balance between bone formation and resorption has shifted such that the rate of bone resorption exceeds bone formation, resulting in net bone loss, trabecular and cortical thinning and porosity (Seeman 2003a).

Periosteum is a dense connective tissue membrane covering the outer surface of all bones, except at the joint of long bones. In both normal and pathological processes the periosteum plays a pivotal role in both bone formation and fracture healing, in conjunction with the involvement of other important factors such as growth factors and 
mechanical loading (Eyre-Brook 1984; Malizos and Papatheodorou 2005; Seeman 2003b). Periosteum is a highly vascularized tissue which contains both osteogenic and chondrogenic progenitor cells, as well as other related bioactive factors (Malizos and Papatheodorou 2005; Nakahara et al. 1990; Simpson 1985). The osteogenic function of periosteum determines the amount of de novo postnatal periosteal bone formation defining the thickness and strength of cortical bone. Previous researchers have revealed an age related structural and cellular degeneration in periosteum (De Bari et al. 2001; Fan et al. 2008; O'Driscoll et al. 2001; Squier et al. 1990; Tonna 1978). However, in osteoporosis, which is characterized by decreased bone strength and a high incidence of fractures, the changes occurring in the periosteum are not well characterized and require further investigation. Although estrogen deficiency is believed to be an intrinsic cause of osteoporosis, the process and mechanism of bone loss in osteoporosis remain unclear. Most researchers have emphasized the bone resorption activity occurring in the endocortical or intramedullary area, but there are no reports in the current literature describing the structural and cellular changes in periosteum of osteoporosis. The purpose of this study is therefore to characterize the structural and cellular differences in both diaphyseal and metaphyseal periosteum of osteoporotic rats.

\section{Materials and methods}

Animal samples and slices

This study was carried out in accordance with the guidelines of the University Animal Ethics Committee. Osteoporosis was induced by ovariectomy of three-months old female Lewis rats, followed by a 30\% caloric reduced diet for 4 months to develop osteoporosis (Xiao et al. 2007). The induced osteoporosis has been confirmed in our previous study (Xiao et al. 2007). Four osteoporotic rats and three normal female Lewis rats (sham-operated group in which ovaries were exposed but only equal volumes of fat tissue was excised), all in 7 months old, were utilized in this experiment. After the animals were sacrificed, the right tibia and femur were retrieved for the following experiments. Tibia were scanned using a Micro CT machine $(\mu \mathrm{CT} 40$, SCANCO Medical AG, Brüttisellen, Switzerland) to confirm the osteoporosis induced in rats. Then both tibia and femur were fixed in $4 \%$ paraformaldehyde for $12 \mathrm{~h}$ at room temperature, then decalcified in 10\% EDTA and embedded in paraffin. Serial sections of sagittal slices, $5 \mu \mathrm{m}$ thick, were cut from the paraffin blocks with a microtome (Leica Microsystems GmbH, Germany). Only slices near the central sagittal plane were used for subsequent experiments.

Structural observation

After Micro CT scanning, the three dimensional (3D) image of trabecular bone in proximal end of tibia from both normal and osteoporotic rats was reconstructed and related histomorphometrical parameters, such as normalized trabecular bone volume (BV/TV), trabecular bone thickness (Tb.Th), trabecular bone separation (Tb.Sp), trabecular bone number (Tb.N) and conectivity density (Conn.D), were calculated by the software package of the Micro CT machine. These images and parameters were compared between two groups to confirm the osteoporosis induced in rats. On the other hand, slices from tibia were stained with hematoxylin and eosin (H\&E) (HD Scientific Supplies, Australia), and the trabecular bones in proximal end were observed under a microscope (Carl Zeiss Microimaging $\mathrm{GmbH}$, Germany) to further confirm the induced osteoporosis.

For periosteum observation, $1 \mathrm{~mm}$ lengths of periosteum from femur diaphysis and $1 \mathrm{~mm}$ lengths of periosteum from proximal femur metaphysis were selected for analysis (Fig. 1). According to the difference of cell and fiber distribution in the periosteum, the cambial and fibrous layers were defined (Augustin et al. 2007). The thickness of fibrous and cambial layers on the middle line perpendicular to the periosteum surface in each microscopic field, as well as the cell number of each layer throughout each periosteal area, were measured using Axion software (Carl Zeiss) under a microscope (Carl Zeiss). Data from each animal of the osteoporotic and normal groups were recorded for further analysis.

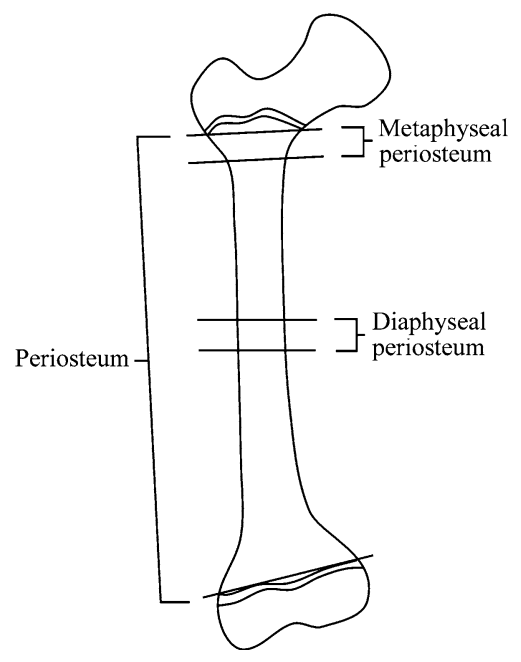

Fig. 1 Illustration of the periosteal sites observed in this study 
Immunohistochemistry

Osteoclasts were identified using a TRAP (tartrate-resistant acid phosphatase) staining kit (Sigma-Aldrich, Australia); ALP (Alkaline Phosphatase) specific antibody (Goat antimouse, Sigma-Aldrich, Australia) was used to identify osteogenic cells; VEGF specific antibody (mouse antihuman, R\&D System, Inc., USA) was used to identify VEGF positive cells; vWF specific antibody (mouse antihuman, Chemicom International Inc., USA) was used to identify blood vessels; TH (Tyrosine Hydroxylase) specific antibody (Rabbit anti-rat, Serotec, UK) was used to identify sympathetic nerve fibers; and CGRP (Calcitonin generelated peptide) specific antibody (Goat anti-rat, Serotec, UK) was used to identify sensory nerve fibers. To validate the results, each experiment was repeated at least three times.

Prior to immunoperoxidase staining, endogenous peroxidase activity was quenched by incubating the tissue sections in $3 \% \mathrm{H}_{2} \mathrm{O}_{2}$ for 20 min. All sections were blocked with $10 \%$ swine serum for $1 \mathrm{~h}$. The enzymatic treatment was used to expose epitopes by incubating the slices with proteinase K (DakoCytomation, USA,) for $10 \mathrm{~min}$ at room temperature. Sections were incubated with optimal dilution of primary antibody ALP (1:300), VEGF (1:300), vWF (1:500), TH (1:400) and CGRP (1:500) at $4^{\circ} \mathrm{C}$ overnight, and the following day incubated for 15 min with a biotinylated swine-anti-mouse, rabbit, goat secondary antibody (DAKO Multilink, USA), followed by $15 \mathrm{~min}$ incubation with horseradish perioxidase-conjugated avidin-biotin complex (ABC). Antibody complexes were visualized after the addition of a buffered diaminobenzidine (DAB) substrate for $4 \mathrm{~min}$; the reaction stopped by immersing and rinsing of sections in PBS. Sections were then lightly counterstained with Mayer's haematoxylin and Scott's Blue for $40 \mathrm{~s}$ each, in between 3 min rinses with running tap water. Following this, the sections were dehydrated with ascending concentrations of ethanol solutions, cleared with xylene and mounted with a cover slip using DePeX mounting medium (BDH Laboratory Supplies, UK). Standard TRAP staining procedure was done according to the manufacturer's instructions.

Controls for the immunohistochemical staining procedures included conditions in which (a) the primary antibody was omitted for staining procedure, and (b) an irrelevant antibody ( $\operatorname{IgG})$, not present in the test sections, was utilized.

At $\times 200$ magnification and with the aid of the AxioVision software, the number of positive cells from each cell population in each periosteal area was counted. Three different slices from each rat from the two groups were observed and the data recorded for subsequent analysis. The number of active $\mathrm{TRAP}^{+}$osteoclasts in bone resorption pits, $\mathrm{ALP}^{+}$and $\mathrm{VEGF}^{+}$cells were normalized against the total cell number and expressed as positive cell number per total of 100 cells from each sample in order to counter the effect that variations of total cell numbers from the two groups had on the positive cell counts. The blood vessel counts were normalized to the vessel number in a $0.03 \mathrm{~mm}^{2}$ periosteal area. As for nerve staining, spatial distribution was observed and described.

\section{Statistical analysis}

The data from both normal and osteoporotic groups were compared using the Student's $t$-test. The data from different periosteum layers and periosteal areas of osteoporotic rats were subjected to one-way ANOVA and SNK tests and results were regarded as significance at $P \leq 0.05$. Data analysis was performed using the SPSS software (SPSS Inc, USA).

\section{Results}

Structural differences between osteoporotic and normal rats

Osteoporotic rat model was further confirmed by the significantly lower trabecular bone volume, thickness, number, connectivity density and higher trabecular bone separation when compared with normal rats $(P<0.05)$ (Table 1, Fig. 2). Typical fenestrated trabeculae were demonstrated in the osteoporotic rat model (Fig. 2).

No statistically significant difference was found in the thickness and cell numbers between osteoporotic and normal rats of the diaphyseal periosteum, except for the thickness of the fibrous layer $(P<0.05)$ (Fig. 3). In the metaphyseal periosteum, osteoporotic rats had thicker and more cellular cambial layers compared with normal rats $(P<0.001)$ (Fig. 3). Although there was no significant difference in the thickness of the fibrous layer $(P>0.05)$, the number of cells in the fibrous layer of osteoporotic rats in the metaphyseal area was higher than normal rats $(P<0.001)$. In osteoporotic rats the metaphyseal periosteum was significantly thicker and more cellular than in the diaphyseal periosteum, especially the cambial layer of metaphyseal periosteum $(P<0.05)$ (Fig. 3).

$\mathrm{TRAP}^{+}$osteoclasts in periosteum

The normalized cell number of active $\mathrm{TRAP}^{+}$osteoclast in bone resorption pits from the cambial layer was used for this analysis. The osteoporotic rats had significantly more active $\mathrm{TRAP}^{+}$osteoclasts in both diaphyseal and metaphyseal periosteal areas $(P<0.001)$ compared to normal 
Table 1 Histomorphometrical comparison of the tibia bone from osteoporotic and normal rats

\begin{tabular}{|c|c|c|c|c|c|}
\hline & $\mathrm{BV} / \mathrm{TV}(\%, x \pm \mathrm{SD})$ & Tb.Th (mm, $x \pm \mathrm{SD})$ & Tb.Sp (mm, $x \pm \mathrm{SD})$ & Tb.N (1/mm, $x \pm \mathrm{SD})$ & Conn.D $\left(1 / \mathrm{mm}^{3}\right)(x \pm \mathrm{SD})$ \\
\hline Osteoporotic* & $6.24 \pm 0.2$ & $0.07 \pm 0.02$ & $1.36 \pm 0.16$ & $0.78 \pm 0.08$ & $15.85 \pm 0.51$ \\
\hline Normal & $20.5 \pm 1.4$ & $0.1 \pm 0.02$ & $0.3 \pm 0.05$ & $3.11 \pm 0.35$ & $51.02 \pm 10.49$ \\
\hline
\end{tabular}

$* P<0.05$

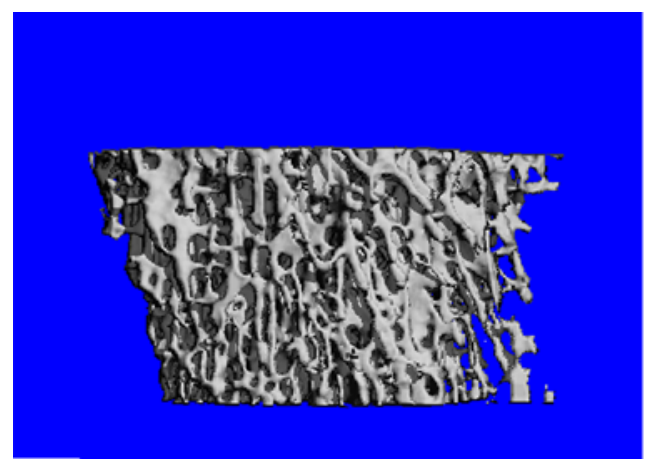

$\mathbf{A}$

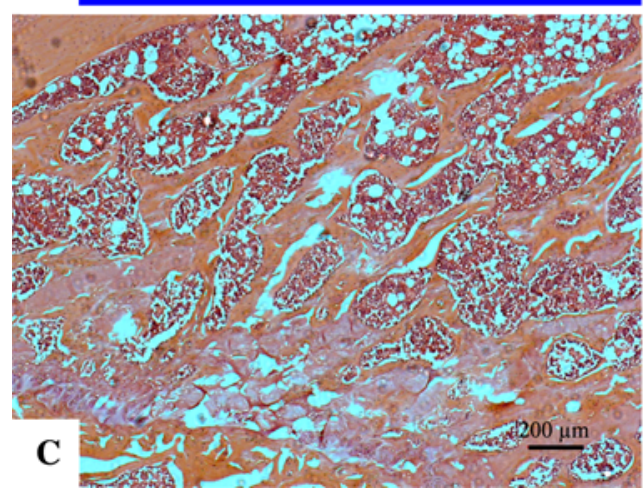

Fig. 2 Histological validation of animal models Osteoporotic rat model was confirmed by the significantly loss of trabecular bones (b) compared with normal rats (a) in 3D reconstructed images; Typical

rats (Fig. 4 and 5). In osteoporotic rats, the cambial layer of metaphyseal periosteum had similar $\mathrm{TRAP}^{+}$osteoclast density in bone resorption pits when compared with the cambial layer of diaphyseal periosteum $(P>0.05)$ (Fig. 4).

\section{ALP expression in periosteum}

The normalized $\mathrm{ALP}^{+}$cell count was used for this analysis. No significant difference was found in the ALP expression in either diaphyseal and metaphyseal periosteum of osteoporotic rats compared to normal rats $(P>0.05)$ (Fig. 4 and 5). No difference was detected between the cambial and fibrous layers in diaphyseal and metaphyseal periosteum, respectively. Nor was there any significant difference
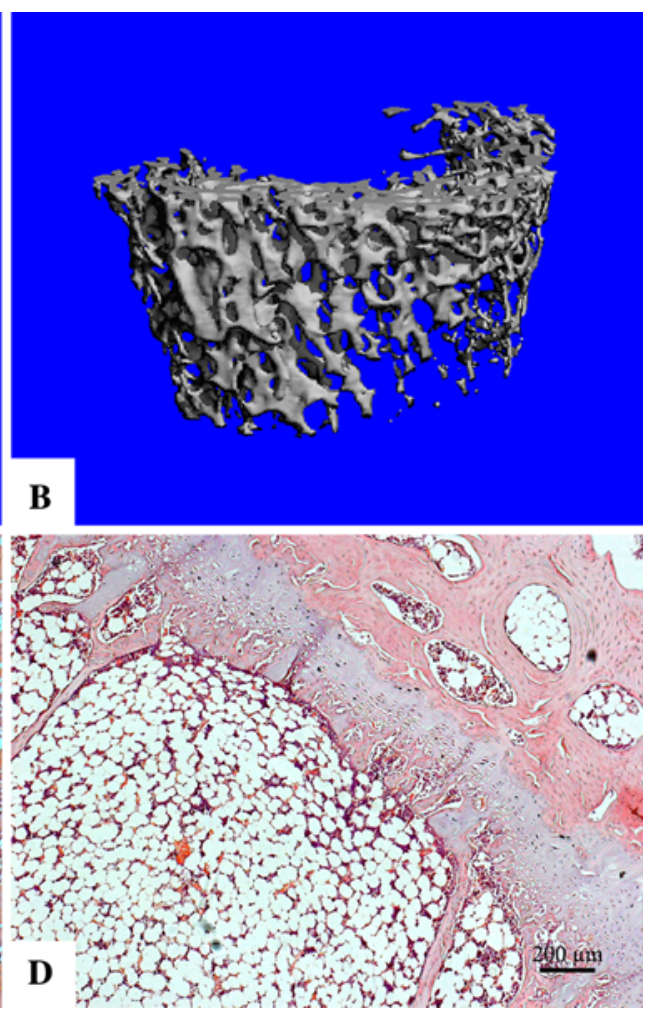

fenestrated trabeculae were demonstrated in the osteoporotic rat model (d) in contrast to normal rats c. $\mathrm{H}$ and $\mathrm{E}$ staining pictures (c and d) were taken at $40 \times$ magnification

to the ALP expression between different periosteal sites in osteoporotic rats $(P>0.05)$ (Fig. 4).

\section{VEGF expression in periosteum}

The normalized $\mathrm{VEGF}^{+}$cell count was used for this analysis. Significantly more $\mathrm{VEGF}^{+}$cells were found in the cambial layer of the metaphyseal periosteum of osteoporotic rats compared to normal rats $(P<0.001)$ (Fig. 4 and 5). No significant difference was found in other periosteal areas. In osteoporotic rats the cambial layer of both the metaphyseal and diaphyseal periosteum had more VEGF positive cells than the fibrous layers in both periosteal areas, whilst the number of VEGF positive cells in the cambial layer of metaphyseal periosteum was higher 
Fig. 3 Diagrams and $\mathrm{H}$ and $\mathrm{E}$ staining $(200 \times)$ of diaphyseal and metaphyseal periosteum from normal and osteoporotic rats. a diaphyseal periosteum from normal rats; $\mathbf{b}$ diaphyseal periosteum from osteoporotic rats; c metaphyseal periosteum from normal rats; $\mathbf{d}$ metaphyseal periosteum from osteoporotic rats. (NO normal rats, $O P$ osteoporotic rats, Dia-c cambial layer of diaphyseal periosteum, Dia-f fibrous layer of diaphyseal periosteum, Meta-c cambial layer of metaphyseal periosteum Meta- $f$ fibrous layer of metaphyseal periosteum; *: $P<0.05$. In pictures: $B$ bone tissue, $P$ periosteum, $C$ cambial layer, $F$ fibrous layer.)
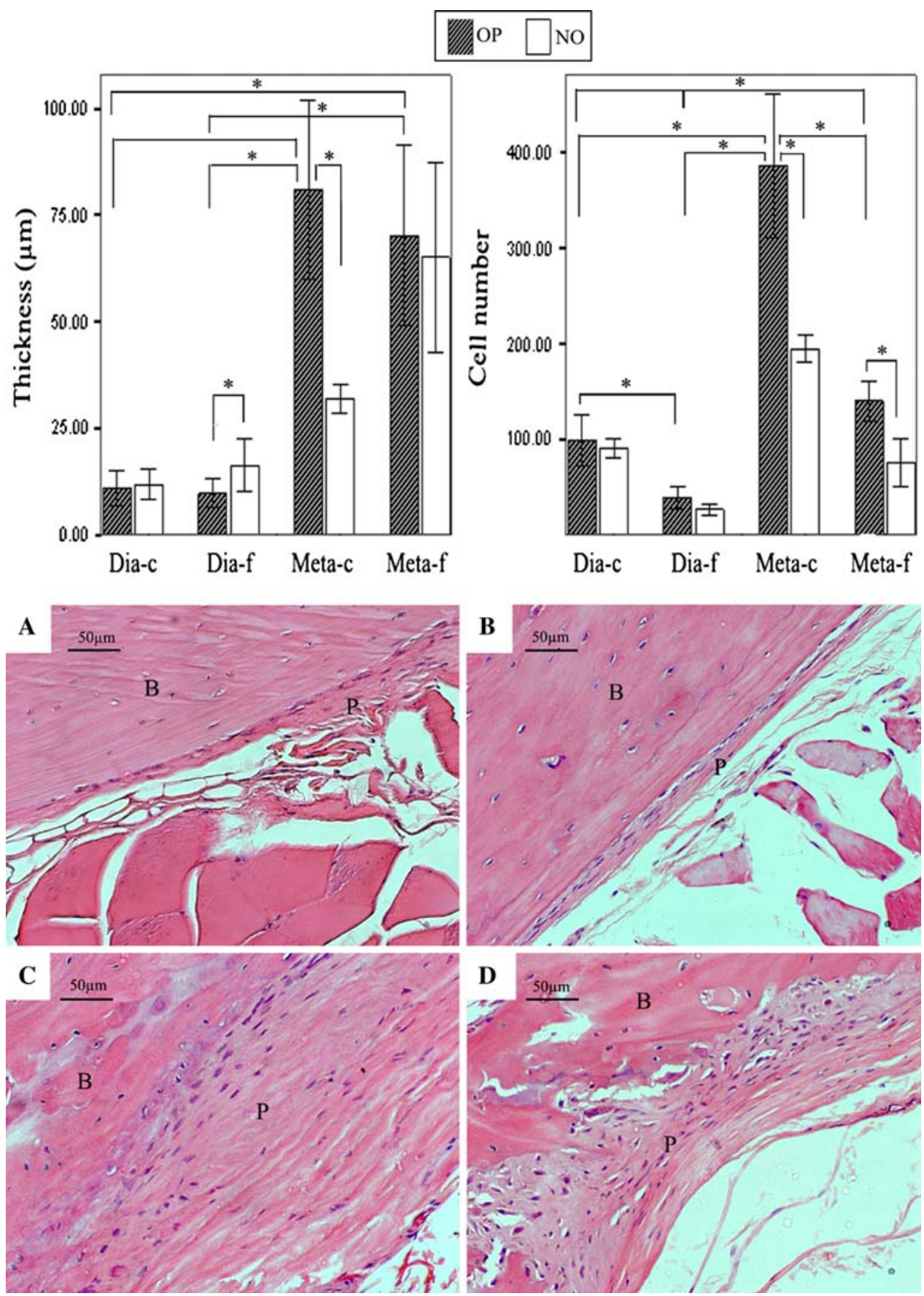

than that in the cambial layer of diaphyseal periosteum $(P<0.001)$ (Fig. 4).

Blood vessels revealed by vWF

The normalized blood vessel count was used for this analysis. Osteoporotic rats had higher degree of vascularization in the cambial layers of both the diaphyseal and metaphyseal periosteum compared to normal rats $(P<0.001)$, especially in the metaphyseal periosteum
(Fig. 4 and 5). No significant difference was found in the fibrous layer of either periosteal area between the two groups. In osteoporotic rats, the cambial layer of metaphyseal periosteum had a higher degree of vascularization than the fibrous layer $(P<0.001)$ (Fig. 4 and 5).

Sympathetic nerves revealed by $\mathrm{TH}$

TH staining revealed that the distribution of sympathetic nerve fibers was mainly present in osteoporotic rats, 


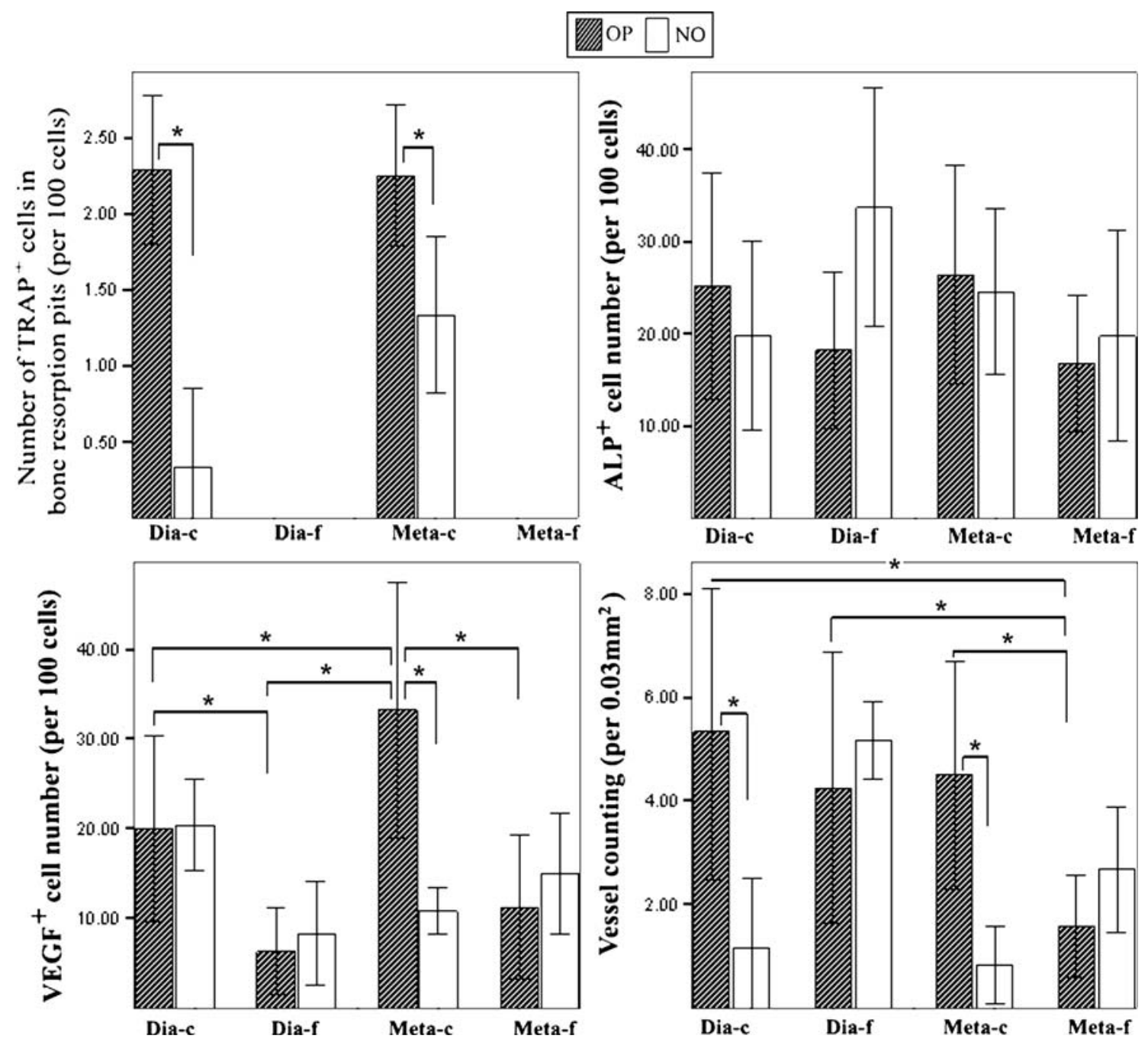

Fig. 4 Cellular differences in diaphyseal and metaphyseal periosteum between osteoporotic and normal rats. (NO normal rats, $O P$ osteoporotic rats, Dia-c cambial layer of diaphyseal periosteum, Dia- $f$

predominantly in the cambial layer of periosteum (Fig. 5). A slight TH stain was observed in the cambial layer of metaphyseal area of normal rat. No obvious staining was detected in the fibrous layer or diaphyseal area.

\section{Sensory nerves revealed by CGRP}

Sensory nerve fibers identified by CGRP antibodies were found in both osteoporotic and normal rats (Fig. 5). There was no discernible difference in the expression of CGRP between the two groups in the different periosteal areas. However, in normal rats $\mathrm{CGRP}^{+}$nerve fibers appeared to be distributed in both fibrous and cambial layers, whereas in osteoporotic rats most $\mathrm{CGFP}^{+}$nerve fibers were present in the cambial layer of the metaphyseal area. fibrous layer of diaphyseal periosteum, Meta-c cambial layer of metaphyseal perios teum, Meta- $f$ fibrous layer of metaphyseal periosteum; *: $P<0.05$ )

\section{Discussion}

Osteoporosis is characterized by a negative balance of bone metabolism which leads to a decreased bone mass with subsequent thinning of the trabecular and cortical bone and a significantly greater risk of bone fractures. The cortical bone expansion through the periosteum is the most effective way to increase bone strength and protect against fractures. Despite the synergistical role and potential significance of periosteum in the pathogenesis and treatment of osteoporosis, little is known about this role of the periosteum in osteoporosis. Knowledge of periosteal structural and cellular characteristics would greatly enhance our understanding of the disorder and aid in the development of more specific and effective treatments.

Osteoporosis patients are at a greater risk of bone fractures, and these fractures have different patterns compared 


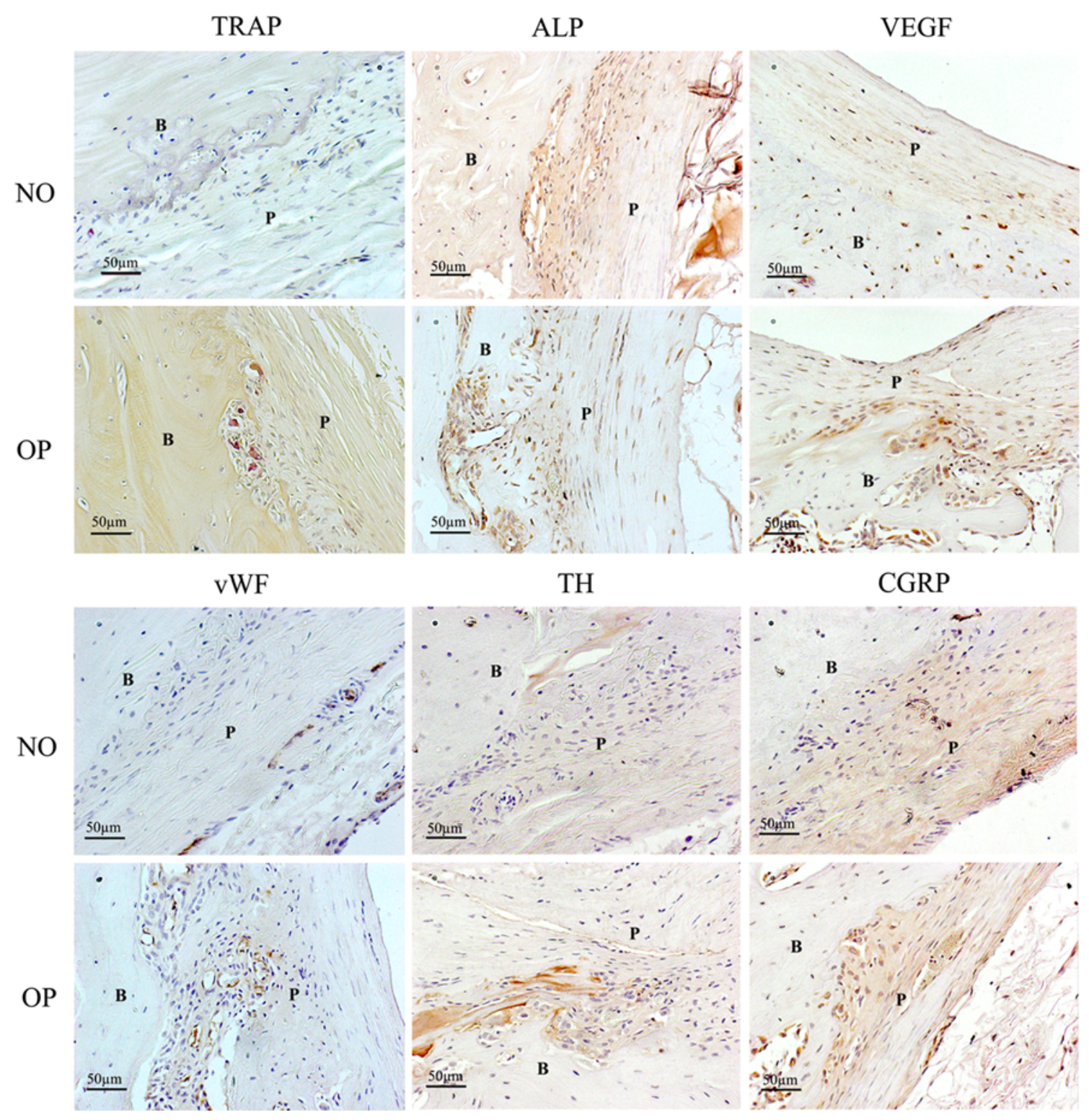

Fig. 5 Immunohistochemical staining $(200 \times)$ of metaphyseal periosteum from normal and osteoporotic rats. (NO normal rats, $O P$ osteoporotic rats, $B$ bone tissue; $P$ periosteum)

with those in patients without osteoporosis (Ferretti et al. 1995). Most fall related fractures in osteoporotic and elderly patients begin in the cortex of the metaphyses or former epiphyses, intertrochanteric, and femoral neck cortices; they never begin in metaphyseal spongiosa and rarely in the diaphyseal cortex (Ferretti et al. 1995). This phenomenon indicates a fundamental difference in the mechanical environment and bone strength along the bone axis. It is known that endosteal or intramedullary bone resorption activity is greater in osteoporotic bone compared to normal bone, which is thought to be the main cause of net bone loss (Parfitt 2002). However, the synergic role of the periosteum in osteoporosis is not well understood. In an attempt to further the understanding of the function of periosteum at different periosteal sites in osteoporosis, we investigated the basic structure and related cell populations in the diaphyseal and metaphyseal periosteum of osteoporotic rats.

In the general H\&E stain and histomorphometry observations, the thickness and cell numbers in the cambial layer of metaphyseal periosteum from osteoporotic rats were found to be much greater compared to normal rats, despite both groups being of similar age ( 7 months). This structural difference appears to be closely related to the different cell populations found in the two cohorts, an observation confirmed by the immunohistochemical staining.

To our knowledge, no previous studies have documented the distribution of active osteoclasts in the 
periosteum covering osteoporotic bones, although periosteal bone turnover activity and osteoclast distribution have recently been documented in the femoral neck in a number of adult rhesus and Japanese macaques (Bliziotes et al. 2006). In our study, periosteal osteoclasts were identified using TRAP specific cell markers. Immunohistochemical observations in this study found the evidence of a noticeable increased fraction of active osteoclasts in the cambial layer of both diaphyseal and metaphyseal periostea from osteoporotic rats. As these observed osteoclasts were found in bone resorption pits on eroded bone surfaces, which means they are active osteoclasts, it might suggest more serious bone resorption activities on both diaphyseal and metaphyseal cortical bone surfaces of osteoporotic rats compared to the normal rats. As the thickness of cortical bone in metaphyseal area is thinner than the diaphyseal area (Furst et al. 2008), the metaphyseal bone could be more susceptible of bone fractures than the diaphyseal in osteoporosis conditions. This phenomenon could potentially have clinical relevance as more fractures are reported in the metaphyseal areas compared to the diaphyseal areas in osteoporotic patients (Ferretti et al. 1995). Age-related bone loss often shows the following morphological changes including decreased periosteal apposition, endosteal bone loss, bone loss on the trabecular, endocortical and intracortical surfaces, although some controversies have been reported on periosteal apposition, which shows that periosteal apposition continues, even after menopause, and may partly offset the bone resorption on the endosteal surface (Ahlborg et al. 2003). However, the findings in our study suggest that bone resorption activity in the periosteum is greater in osteoporotic rats compared to normal rats and that the behavior of the osteoclast cells is destructive. On the other hand, osteogenic activity demonstrated by the ALP expression in this experiment revealed no significant difference was detected in either the different periosteal sites of osteoporotic rats or between the two groups of rats. Given the increased number of active osteoclasts in the periosteum of osteoporotic rats, the relatively stable osteogenic activity might therefore be inadequate to offset the bone resorption, which would consequently incur the net bone loss and strength decrease. This failed adaptation of bone to the bone resorption could be the result of the estrogen withdrawal in the osteoporotic rats since the estrogen is believed to be able to preserve the bone mass and decrease the bone resorption activity (Blahos 2007; Kameda et al. 1997). According to these findings, periosteum seems playing more destructive roles in osteoporosis, and new periosteum-targeting medicines or operations could be developed to suppress the bone resorption and enhance the osteogenic activities in periosteum, especially in metaphyseal area.
VEGF is an important growth factor which is reported to be involved in angiogenic, osteogenic and osteoclastogenic processes (Cho et al. 2005; Nakagawa et al. 2000; Peng et al. 2005). Bone development, angiogenesis, bone formation and bone resorption are all closely associated processes with VEGF known to stimulate osteoclast activity (Cho et al. 2005; Nakagawa et al. 2000; Peng et al. 2005). Osteoclast activity and angiogenesis may therefore be regulated by a common mediator such as VEGF. The data in our study seems to support this view since a higher expression of VEGF, as well as more $\mathrm{vWF}^{+}$blood vessels, was found in the vicinity of active $\mathrm{TRAP}^{+}$osteoclasts in osteoporotic rats compared to normal rats. The increase of VEGF expression and periosteal vascularization in the periosteum of the osteoporotic rats may be related to the increased bone resorptive activity in these periosteal areas.

The reason for the increased expression of sympathetic nerve fibers in the periosteum in osteoporosis is unclear, although it is well documented that sympathetic nerve fibers are present in both the periosteum and bone (Hill et al. 1991a; Hohmann et al. 1986). Sympathetic innervation of periosteum and bone contributes to the regulation of bone cell activity and, therefore, the eqiulibrium between bone modeling and remodeling (de Souza et al. 2005). It is known that surgical and chemical sympathectomy can both modulate bone cell function. However, the sympathetic nervous system (SNS) can give rise to both anabolic and catabolic effects (Cherruau et al. 1999; Haug et al. 2003; Hill et al. 1991b; Sherman and Chole 2000) and its role in regulating bone remodeling is, therefore, controversial. In this study, the distribution of sympathetic nerve fibers was demonstrated by employing an antibody against an SNS specific marker, tyrosine hydroxylase, which is expressed in adrenergic fibers. More sympathetic nerve fibers were found in the cambial layer of periosteum of osteoporotic rats compared to normal rats and this finding was also in accordance with the distribution of $\mathrm{TRAP}^{+}, \mathrm{ALP}^{+}$, $\mathrm{VEGF}^{+}$cells and blood vessels. The co-existence of nerve fibers and various cell populations indicates that sympathetic nerves in the periosteum are involved in the pathogenesis of osteoporosis by interacting with, and possibly regulating, osteogenic, osteoclastogenic and angiogenic factors. Sensory nerve fibers identified by CGRP antibody were found similarly expressed in both osteoporotic and normal rats, although the distribution is slightly different between the two groups. It is generally accepted that CGRP can promote osteogenesis and decrease bone resorption (Cherruau et al. 2003; Imai and Matsusue 2002). CGRP has also been shown to inhibit osteoclastic resorption like calcitonin (Imai and Matsusue 2002). The similar expression of CGRP in both osteoporotic and normal rats suggests a similar osteogenesis-related role for CGRP in the two groups. 
Based on the findings from this study, it could be concluded that the periosteum of osteoporotic rats differs from normal rats both in terms of structure and cell populations. This is especially evident in the cambial layer of metaphyseal periosteum. Bone resorption appears to be more active in the periosteum of osteoporotic rats compared to normal rats, whereas bone formation activity is comparable between the two groups. The dysregulation of bone resorption and formation in the periosteum, characteristic of the pathogenesis of osteoporosis, may therefore be the effect of the interaction between various neural pathways and the cell populations residing within it. The findings of this study were observed from rat osteoporosis model. It is known that there are some differences between human and rat in bone remodelling and fragility due to the differences in bone biology and physiology, therefore, these findings need to be further validated in mammal models to be relevant to human osteoporosis.

Acknowledgments: We would like to thank Ms Wei Shi for her technique support in immunohistochemical work and Mr. Thor Friis for his proof reading of the entire manuscript. The Stichting Nijmeegs University Funds (SNUF) and the Reumastichting provided financial support for the internship of Stefan AW Bouwense. Wei Fan is on a Faculty Scholarship from the Queensland University of Technology.

Open Access This article is distributed under the terms of the Creative Commons Attribution Noncommercial License which permits any noncommercial use, distribution, and reproduction in any medium, provided the original author(s) and source are credited.

\section{References}

Ahlborg HG, Johnell O, Turner CH, Rannevik G, Karlsson MK (2003) Bone loss and bone size after menopause. N Engl J Med 349:327-334

Augustin G, Antabak A, Davila S (2007) The periosteum. Part 1: anatomy histology and molecular biology. Injury 38:11151130

Blahos J (2007) Treatment and prevention of osteoporosis. Wien Med Wochenschr 157:589-592

Bliziotes M, Sibonga JD, Turner RT, Orwoll E (2006) Periosteal remodeling at the femoral neck in nonhuman primates. J Bone Miner Res 21:1060-1067

Cherruau M, Facchinetti P, Baroukh B, Saffar JL (1999) Chemical sympathectomy impairs bone resorption in rats: a role for the sympathetic system on bone metabolism. Bone 25:545-551

Cherruau M, Morvan FO, Schirar A, Saffar JL (2003) Chemical sympathectomy-induced changes in TH-, VIP-, and CGRPimmunoreactive fibers in the rat mandible periosteum: influence on bone resorption. J Cell Physiol 194:341-348

Cho SW, Lim SH, Kim IK et al (2005) Small-diameter blood vessels engineered with bone marrow-derived cells. Ann Surg 241:506515

De Bari C, Dell'Accio F, Luyten FP (2001) Human periosteumderived cells maintain phenotypic stability and chondrogenic potential throughout expansion regardless of donor age. Arthritis Rheum 44:85-95 de Souza RL, Pitsillides AA, Lanyon LE, Skerry TM, Chenu C (2005) Sympathetic nervous system does not mediate the load-induced cortical new bone formation. J Bone Miner Res 20:2159-2168

Eyre-Brook AL (1984) The periosteum: its function reassessed Clin Orthop Relat Res: 300-307

Fan W, Crawford R, Xiao Y (2008) Structural and cellular differences between metaphyseal and diaphyseal periosteum in different aged rats. Bone 42:81-89

Ferretti JL, Frost HM, Gasser JA et al (1995) Perspectives on osteoporosis research: its focus and some insights from a new paradigm. Calcif Tissue Int 57:399-404

Furst A, Meier D, Michel S, Schmidlin A, Held L, Laib A (2008) Effect of age on bone mineral density and micro architecture in the radius and tibia of horses: an Xtreme computed tomographic study. BMC Vet Res 4:3

Haug SR, Brudvik P, Fristad I, Heyeraas KJ (2003) Sympathectomy causes increased root resorption after orthodontic tooth movement in rats: immunohistochemical study. Cell Tissue Res 313:167-175

Hill EL, Elde R (1991) Distribution of CGRP-, VIP-, D beta H-, SP-, and NPY-immunoreactive nerves in the periosteum of the rat. Cell Tissue Res 264:469-480

Hill EL, Turner R, Elde R (1991) Effects of neonatal sympathectomy and capsaicin treatment on bone remodeling in rats. Neuroscience 44:747-755

Hohmann EL, Elde RP, Rysavy JA, Einzig S, Gebhard RL (1986) Innervation of periosteum and bone by sympathetic vasoactive intestinal peptide-containing nerve. Fibers Science 232:868-871

Imai S, Matsusue Y (2002) Neuronal regulation of bone metabolism and anabolism: calcitonin gene-related peptide-, substance P-, and tyrosine hydroxylase-containing nerves and the bone. Microsc Res Tech 58:61-69

Kameda T, Mano H, Yuasa T et al (1997) Estrogen inhibits bone resorption by directly inducing apoptosis of the bone-resorbing osteoclasts. J Exp Med 186:489-495

Malizos KN, Papatheodorou LK (2005) The healing potential of the periosteum molecular aspects. Injury 36(Suppl 3):S9-S13

Nakagawa M, Kaneda T, Arakawa T et al (2000) Vascular endothelial growth factor (VEGF) directly enhances osteoclastic bone resorption and survival of mature osteoclasts. FEBS Lett 473:161-164

Nakahara H, Bruder SP, Haynesworth SE et al (1990) Bone and cartilage formation in diffusion chambers by subcultured cells derived from the periosteum. Bone 11:181-188

O'Driscoll SW, Saris DB, Ito Y, Fitzimmons JS (2001) The chondrogenic potential of periosteum decreases with age. J Orthop Res 19:95-103

Parfitt AM (2002) Size of bone in the aged endocortical resorption. J Bone Miner Res 17:1306 (author reply 7-8)

Peng H, Usas A, Olshanski A et al (2005) VEGF improves, whereas sFlt1 inhibits, BMP2-induced bone formation and bone healing through modulation of angiogenesis. J Bone Miner Res 20:20172027

Pogoda P, Priemel M, Rueger JM, Amling M (2005) Bone remodeling: new aspects of a key process that controls skeletal maintenance and repair. Osteoporos Int 16(Suppl 2):S18-S24

Rauch F, Travers R, Glorieux FH (2007) Intracortical remodeling during human bone development-a histomorphometric study. Bone 40:274-280

Seeman E (2003a) Invited review: pathogenesis of osteoporosis. J Appl Physiol 95:2142-2151

Seeman E (2003b) Periosteal bone formation-a neglected determinant of bone strength. N Engl J Med 349:320-323

Seeman E (2003c) Reduced bone formation and increased bone resorption: rational targets for the treatment of osteoporosis. Osteoporos Int 14(Suppl 3):S2-S8 
Sherman BE, Chole RA (2000) Sympathectomy, which induces membranous bone remodeling, has no effect on endochondral long bone remodeling in vivo. J Bone Miner Res 15:1354-1360

Simpson AH (1985) The blood supply of the periosteum. J Anat 140(Pt 4):697-704

Squier CA, Ghoneim S, Kremenak CR (1990) Ultrastructure of the periosteum from membrane bone. J Anat 171:233-239
Tonna EA (1978) Electron microscopic study of bone surface changes during aging. The loss of cellular control and biofeedback. J Gerontol 33:163-177

Xiao Y, Fu H, Prasadam I, Yang YC, Hollinger JO (2007) Gene expression profiling of bone marrow stromal cells from juvenile, adult, aged and osteoporotic rats: with an emphasis on osteoporosis. Bone 40:700-715 